\title{
LA NATURA ANALOGICA DEL BENE: RADICE METAFISICA DELLA LIBERTÀ DELLA SCELTA IN TOMMASO D'AQUINO
}

\author{
Federica Bergamino \\ Pontificia Università della Sanła Croce (Roma)
}

\section{RESUMEN}

L'articolo vuole evidenziare come l'atto del libero arbitrio in Tommaso d'Aquino non si realizzi semplicemente nella scelta tra il bene e il male, ma più essenzialmente esso consiste nella scelta libera del meglio. Ruolo chiave nella disamina la concezione metafisica tommasiana del bene e il rapporto di esso con il soggetto libero. Una volta evidenziata la speciale causalità del bene -e in concreto del bene particolare-si esamina la scelta alla luce della natura analogica del «meglio».

Parole chiave: Libertà, scelta, causalità del bene, analogia, meglio.

\begin{abstract}
The paper aims to show how the act of free will in Thomas Aquinas is not exercised merely in the choice between good an evil, but consists more essentially in the free choice of the better. What plays the key role in the analysis is Thomas's metaphysical conception of the good and its relationship to the free subject. The special causality of the good - and more concretely, of the particular good - is noted, and then choice is examined in light of the analogical nature of the «better».
\end{abstract}

Key words: Freedom, choice, causality of the good, analogy, better.

«Chi dice libero arbitrio, dice indifferenza (...). La libertà è un caso privilegiato di indifferenza attiva. (...) La volontà, al termine della deliberazione, può volere e non volere. Il suo privilegio tra le cause indifferenti, consiste nel dominio che essa esercita sulla forza attrattiva delle proprie operazioni: è da essa stessa che viene, in fin dei conti, la loro attrazione, contemporaneamente all'attrazione del loro oggetto»' 1 .

È comune in ambito tomista, rinvenire una spiegazione della libertà in termini di indifferenza attiva rispetto ai beni particolari ${ }^{2}$. È altrettanto comune però che poi sorga la domanda circa il motivo delle nostre scelte ${ }^{3}$ : perché si sceglie una cosa piuttosto che un'altra se i beni sono uguali, se c'è indifferenza dell'uno rispetto all'altro?

1 SIMON, Y., Trattato del libero arbitrio, Paoline, Roma 1957, p. 138; tit. orig. Traité du libre arbitre, Liège, Paris 1951.

2 Ho in mente qui una linea interpretativa dominante del tomismo del ' 900 che da Garrigou-Lagrange, arriva fino a Gallagher. Tra gli autori di riferimento in tale posizione mi limito a menzionare: Sertillanges, Gorce, Browne, Maritain Manser, Lottin, Simon, De Finance, Vanni Rovighi.

3 Cfr. al proposito GARRIGOU-LAGRANGE, R., Intellectualisme et liberté chez Saint Thomas, «Revue des sciences philosophiques et théologiques», II parte (1908), p. 15. 
A mio avviso l'esegesi tomista, anche nei suoi autori più recenti ${ }^{4}$, non offre una risposta adeguata alla questione. La soluzione del problema così come si configura, risalta il primato attivo del volere, ma lasciando irrisolta la questione della ragionevolezza della scelta. Come si legge sopra, l'attrazione delle operazioni del volere, così come del loro oggetto, viene dal volere stesso. $O$ detto in altri termini: non c'è un motivo della scelta che non sia il volere stesso. La scelta è - in parole di Garrigou-Lagrange — «la risposta gratuita sorta dalle profondità infinite della volontà alla sollecitazione impotente di un bene finito ${ }^{5}$. Non ci sarebbe quindi un movente estrinseco al volere stesso nell'atto di scelta, e il bene particolare non avrebbe alcuna forza causale. Chi dice libero arbitrio dice pertanto indifferenza.

Ora, effettivamente nella concezione di Tommaso d'Aquino la volontà è tendenza desiderativa intellettuale. Per questo è aperta al bene in tutta la sua universalità e necessariamente attratta solo dal bene totale, da quel bene che si presenta come bene in tutti gli aspetti che si possono considerare ${ }^{6}$. Tale apertura universale della volontà non la vincola necessariamente a nessun bene particolare che si presenti come tale. Per questo egli afferma una certa indifferenza attiva del volere che si può portare indifferentemente verso molti beni senza essere attratto in modo necessario da nessuno di essi ${ }^{7}$.

Penso tuttavia che in Tommaso si troverebbe una certa avversione per un'assimilazione tout court del libero arbitrio con l'indifferenza (anche se si tratta di indifferenza attiva). Si legge invero in un'obiezione alla libertà della scelta:

se ci sono due cose del tutto uguali, uno non può sentirsi spinto più verso l'uno che verso l'altro: un affamato per es. che avesse del cibo ugualmente appetibile in direzioni opposte, e a uguale distanza, non si muoverebbe né verso l'una né verso l'altra direzione (...). D'altra parte la scelta di un bene minore è anche meno possibile che quella di un bene uguale. Perciò se vengono proposte due o più cose, tra le quali una pare di maggior valore, è impossibile l'elezione delle altre. Dunque si sceglie per necessità la cosa che appare di maggior valore. Ma ogni scelta ha precisamente per oggetto il mezzo che in qualche modo si presenta migliore. Dunque ogni elezione è imposta da una necessità ${ }^{8}$.

L'obiezione è oltremodo interessante: da una parte si rileva che tra due cose uguali in quanto tali la scelta è impossibile, e pertanto sembra che l'oggetto di scelta non debba essere «indifferente» al volere; dall'altra si pone il problema che se tra due cose una è migliore dell'altra, è impossibile che la scelta sia libera, perché il bene maggiore attrae necessariamente la volontà. Ciò che però sembra di maggiore interesse nell'obiezione, e come si vedrà nella risposta, è l'affermare il criterio del meglio come strutturale alla scelta stessa: sembra infatti che ogni scelta abbia precisamente per oggetto il mezzo che in qualche modo si presenta migliore. D'altra parte per Tommaso è un punto indubbio che chi agisce cerca di agire sempre per il meglio,

4 Cfr. GALLAGHER, D. M., Free Choice and Free Jidgment in Thomas Aquinas, «Archiv fur Geschichte der Philosophie», 76 (1994), pp. 247-277.

5 GARRIGOU-LAGRANGE, (1908), p. 26.

6 Cfr. de malo, q. 6 a un.

7 Cfr. S. Th., I-II q. 10 a. 4: «quia igitur voluntas est activum principium non determinatum ad unum, sed indifferenter se habens ad multa».

8 S. Th., I-II q. 13 a.6 ob.3: «praeterea, si aliqua duo sunt penitus aequalia, non magis movetur homo ad unum quam ad aliud, sicut famelicus, si habet cibum aequaliter appetibilem in diversis partibus, et secundum aequalem distantiam, non magis movetur ad unum quam ad alterum, ut plato dixit, assignans rationem quietis terrae in medio, sicut dicitur in ii de caelo. sed multo minus potest eligi quod accipitur ut minus, quam quod accipitur ut aequale. Ergo si proponantur duo vel plura, inter quae unum maius appareat, impossibile est aliquod aliorum eligere. Ergo ex necessitate eligitur illud quod eminentius apparet. Sed omnis electio est de omni eo quod videtur aliquo modo melius. ergo omnis electio est ex necessitate». 
Infatti, è evidente che l'uomo imita, cioè desidera ciò che partecipa più della bontà, e ciò che è migliore; ma giudichiamo sempre il migliore in rapporto a una misura e per questo motivo dobbiamo accogliere una misura quando deliberiamo che cosa si deve fare preferibilmente. Questo il mezzo con cui la ragion pratica sillogizza che cosa dobbiamo scegliere'.

Ora, all'obiezione sopra citata Tommaso risponde che

nulla impedisce quando sono proposte due cose uguali sotto un medesimo aspetto, che si consideri per una di esse qualche condizione per la quale emerge e che quindi la volontà pieghi più verso l'una che verso l'altra ${ }^{10}$.

Si mette così in evidenza che per scegliere bisogna considerare una qualche condizione per cui la cosa si presenta migliore rispetto a quella non scelta, e pertanto la posizione di Tommaso risulta in linea con l'obiettore per il quale l'oggetto della scelta è precisamente il mezzo migliore, o più precisamente quello che presenta condizioni migliori. Contrariamente all'obiettore però, e questo è il punto chiave del problema, l'Aquinate non vede un conflitto tra la scelta del meglio e la libertà. Per Tommaso si sceglie liberamente il mezzo migliore.

Ora, questo è precisamente ciò che vorrei mettere in evidenza nelle pagine che seguono: la scelta libera del meglio. Vorrei infatti mostrare come nella concezione di Tommaso non solo non c'è incompatibilità tra libertà e scelta del meglio, ma che la libertà si esercita precisamente nella scelta del meglio.

Il punto mi sembra interessante da evidenziare in quanto, come si accennava all'inizio, l'esegesi tomista sembra non rendere sufficientemente ragione di ciò. Infatti, la posizione di chi legge la libertà in Tommaso come mera indifferenza attiva, intesa come primato assoluto del volere sull'oggetto, rende automaticamente impossibile la scelta del meglio: se la volontà nello scegliere è indifferente rispetto ai diversi beni particolari, che senso ha parlare di un meglio, ossia di un'emergenza dell'uno rispetto all'altro?

Si tratta quindi di esaminare gli scritti dell'Aquinate per vedere in che cosa consiste la scelta libera del meglio e quali ne sono le condizioni di possibilità. A mio avviso è parte essenziale della spiegazione della libertà in Tommaso la sua concezione metafisica del bene e il rapporto di questo con il soggetto libero. A motivo di ciò penso di evidenziare in primo luogo la causalità del bene -e in concreto del bene particolare - per poi affrontare la scelta e la natura del meglio.

\section{IL BENE E IL PRINCIPIO DELL'AZIONE}

Nella questione VI del De malo, dedicata allo studio sulla scelta, la dimostrazione del respondeo inizia con un parallelismo tra le cose naturali e l'uomo: come in tutte le altre cose c'è un principio dell'agire così anche negli uomini.

Questo principio attivo o movente negli uomini è propriamente l'intelletto e la volontà, come si dice in III De anima. Tale principio in parte conviene e in parte differisce dal

9 In de anima, L. 3, lect. $16 \S 6$ : «manifestum est enim quod homo, imitatur, idest desiderat, id quod est magis in bonitate, et id quod est melius: melius autem semper diiudicamus aliqua mensura: et ideo oportet accipere aliquam mensuram in deliberando quid magis sit agendum. et hoc est medium ex quo ratio practica syllogizat quid sit eligendums.

10 S. Th., I-II q. 13 a. 6 ad 3: «ad tertium dicendum quod nihil prohibet, si aliqua duo aequalia proponantur secundum unam considerationem, quin circa alterum consideretur aliqua conditio per quam emineat, et magis flectatur voluntas in ipsum quam in aliuds. 
principio attivo delle cose naturali. Conviene certamente, perché come nelle cose naturali si trova la forma che è principio dell'azione, e l'inclinazione che segue alla forma che è detto appetito naturale da cui segue l'azione; così nell'uomo si trova la forma intellettiva e l'inclinazione della volontà che consegue alla forma appresa, da cui deriva l'atto esterno; ma in ciò sta la differenza, che la forma della cosa naturale è una forma individuata dalla materia; quindi l'inclinazione che ne consegue è determinata ad un'unica cosa, mentre la forma intellettuale è universale, e può comprendere molte cose all'interno di essa. Quindi poiché gli atti sono nelle cose singolari nelle quali nulla è adeguato alla potenza universale, resta che l'inclinazione della volontà è aperta indeterminatamente a molte cose ${ }^{11}$.

Qui, come premessa e prima parte della dimostrazione della libertà della scelta, si sottolinea un punto centrale: il fatto che ogni agente, sia esso puramente naturale (fisico) o intelligente, possiede un'inclinazione ad agire, ossia una tendenza. $\mathrm{E}$ la possiede in virtù di una forma. L'inclinazione segue la forma. Questa forma quindi è un principio dell'agire, è «in se stessa una fonte della sua inclinazione ad agire ${ }^{12}$. Dire forma - spiega infatti Tommaso- è dire atto: la forma è atto in quanto è il principio e il fine dell'azione ${ }^{13}$, e «ogni agente agisce in forza della sua formas ${ }^{14}$.

Ora, ci si può chiedere, in che senso la forma è il principio e il fine dell'azione. La messa in evidenza di alcuni elementi inerenti il rapporto fine-forma può aiutare a chiarire il ruolo della nozione di forma, nonché del bene.

Il fine dell'atto costituisce propriamente l'oggetto dell'inclinazione che è il bene ${ }^{15}$. Il bene è invero ciò che tutti desiderano, è in se stesso oggetto di appetito o desiderio ${ }^{16}$, è la spiegazione stessa del desiderio ${ }^{17}$; infatti si comprende un desiderio, ossia, si coglie che ha un senso, solo quando si capisce la bontà del suo oggetto. Il bene è invero la perfezione che conviene a ogni $\operatorname{cosa}^{18}$, e la perfezione è intrinsecamente adeguata al desiderio; è quindi propriamente un oggetto di inclinazione, che ha carattere di causa finale ${ }^{19}$. Ogni fine è infatti qualche tipo di bene, e ogni bene qualche tipo di fine, ma le due nozioni non coincidono esattamente. La no-

11 De malo, q. 6 a. un.: «hoc autem activum sive motivum principium in hominibus proprie est intellectus et voluntas, ut dicitur in III De anima. Quod quidem principium partim convenit cum principio activo in rebus naturalibus, partim ab eo differt. Convenit quidem, quia sicut in rebus naturalibus invenitur forma, quae est principium actionis, et inclinatio consequens formam, quae dicitur appetitus naturalis, ex quibus sequitur actio; ita in homine invenitur forma intellectiva, et inclinatio voluntatis consequens formam apprehensam, ex quibus sequitur exterior actio: sed in hoc est differentia, quia forma rei naturalis est forma individuata per materiam; unde et inclinatio ipsam consequens est determinata ad unum, sed forma intellecta est universalis sub qua multa possunt comprehendi; unde cum actus sint in singularibus, in quibus nullum est quod adaequet potentiam universalis, remanet inclinatio voluntatis indeterminate se habens ad multa).

12 BROCK S.L., Action and Conduct. Thomas Aquinas and the Theory of Action, T\&T Clark, Edinburgh 1998, p.117.

13 Cfr. De pot. q. 1 a.1.

$14 S . T h .$, I q. 3 a. 2.

15 Cfr. $S . T h .$, I q.5 a.4.

16 Cfr. $S$. Th., I q. 5 a. 1. Anche $S$. Th., I-II q. 8 a. 1: «Omnis autem appetitus non est nisi boni. Cuius ratio est quia appetitus nihil aliud est quam inclinatio appetentis in aliquid. Nihil autem inclinatur nisi in aliquid simile et conveniens. Cum igitur omnis res, inquantum est ens etica substantia, sit quoddam bonum, necesse est ut omnis inclinatio sit in bonum. Et inde est quod Philosophus dicit in I Ethic. [c.1, lect. 1], quod bonum est "quod omnia appetunt"》.

17 Il desiderio non è altro che un movimento ordinato al bene.

18 Cfr. S. Th., I q.5 a.5: «Ad formam autem consequitur inclinatio àd finem, aut ad actionem, aut ad aliquid huiusmodi: quia unumquodque, inquantum est actu, agit, et tendit in id quod sibi convenit secundum sua formam».

19 Cfr. S. Th., I q. 5 a. 4. 
zione di bene aggiunge infatti a quella di fine la spiegazione del suo essere un fine ${ }^{20}$. Propriamente parlando quindi ciò che muove all'azione e la spiega nella sua radicalità è il bene.

Ora, la forma è propriamente ciò per cui l'agente viene messo in rapporto con l'oggetto dell'inclinazione, il bene; infatti la convenienza del bene alla cosa è precisamente secondo la forma della cosa. E questo equivale a dire che la forma è principio di inclinazione: costituisce infatti la modalità in cui il bene è presente nell'agente, e quindi ciò in virtù di cui l'agente tende verso di esso.

Pertanto ciò in cui consiste il fine, per costituire il fine dell'atto deve corrispondere alla forma che sta nell'agente, ossia l'agente e il fine dell'agente devono coincidere «in forma». L'oggetto dell'inclinazione per essere tale deve infatti essere presente in qualche modo nell'agente stesso. Ed è proprio in quanto nell'agente si dà tale forma, ossia l'ordine al fine intrinseco all'agente stesso, che l'agente tende e può agire per il fine ${ }^{21}$. L'agente agisce quindi non solo «a causa di un'inclinazione, ma è inclinato ad agire a causa dell'oggetto stesso della sua inclinazione, nella misura in cui l'oggetto gli si presenta in un qualche modo ${ }^{22} \gg$, in una qualche forma. Per questo la forma è principio dell'agire, perché è mediatrice della causalità del fine, ciò attraverso cui il fine suscita inclinazione. $E$ «il fine non è solo un principio della specificazione dell'atto, ma è anche un principio del suo esercizio ${ }^{23}$; l'oggetto dell'inclinazione è causa della causalità dell'agente ${ }^{24}$.

Si può sottolineare il fatto che l'oggetto non inclina l'agente nel modo di un agente che agisce su di esso; piuttosto, ha un genere di causalità a sé, il genere richiamato quando qualcosa è spiegata dal fatto che è buono o migliore. Non prende il posto della causalità agente; piuttosto spiega la causalità dell'agente stesso ${ }^{25}$.

La causa finale pertanto - il bene- «è la causa della causalità efficiente perché fa sì che l'efficiente sia efficientes ${ }^{26}$; ne è quindi la spiegazione ultima e più radicale. Detto primato della causa finale dimostra quindi che la forma - presentando il bene- è veramente il principio dell'agire. Pertanto nel momento in cui l'agente ha la forma per agire, esso può agire.

Ora, nel caso specifico del soggetto umano, dotato di volontà, si rileva che la forma di origine e dell'essere dell'atto volitivo è intellettuale. La volontà vuole il bene conosciuto dall'intelletto. Nel caso dell'agente intellettuale quindi la causalità del bene avviene attraverso l'intelletto ${ }^{27}$. L'intelletto infatti coglie la bontà in se stessa, ossia la vera forma del bene; la forma intellettiva che sta alla radice dell'atto volitivo consiste così nella ragione stessa di bene. Tale forma quindi è universale, e in essa rientrano molte cose particolari. Anzi, molte forme particolari di bene. Più avanti si considererà il rapporto che queste forme hanno tra di loro; sarà alla

20 Cfr. C. G., L. III, c. 3: «Inde enim manifestum est omne agens agere propter finem, quia quodlibet agens tendit ad aliquod determinatum. Id autem ad quod agens determinate tendit, oportet esse conveniens ei: non enim tenderet in ipsum nisi propter aliquam convenientiam ad ipsum. Quod autem est conveniens alicui, est ei bonum. Ergo omne agens agit propter finems.

21 Cfr. Ibidem.

22 BROCK (1998), p. 117.

23 Ibidem; per la duplice valenza della causalità del fine si veda anche DE FINANCE J., Saggio sull'agire umano, Libreria editrice Vaticana, Roma 1992, pp. 67-70; tit. orig. Essai sur l'agir humain, Presses de l'Université Grégorienne, Roma 1962.

24 Cfr. DE FINANCE (1992), p. 69: «la causa è veramente efficiente soltanto mediante la sua ordinazione al fine. Il fine è la causa della causalità della causa».

25 BROCK (1998), p. 118.

26 De principiis naturae, c. 4: «Finis est causa causalitatis efficientis, quia facit efficientem esse efficientem».

27 L'intelletto è infatti causa non solo dell'origine dell'atto di volontà, ma essendone causa formale, presentando l'oggetto, anche del suo stesso essere. Si ha infatti un atto di volontà mentre si sta considerando un oggetto $\mathrm{e}$ mentre si sta considerando che cosa sia volere il suo oggetto. 
luce di questo rapporto che si potrà risolvere la questione del «meglio». Prima però conviene esaminare il modo in cui dette forme particolari possano essere causa dell'atto volitivo.

\section{LA CAUSALITÀ DEL BENE PARTICOLARE}

Un testo di Tommaso è particolarmente esplicito al riguardo. Si legge:

la forma appresa è principio movente in quanto è appresa sotto la ragione di bene o di conveniente: poiché l'atto esterno negli esseri che muovono se stessi deriva dal giudizio col quale una cosa è giudicata buona o conveniente mediante la forma suddetta. Se quindi chi giudica applica se stesso a giudicare, bisogna che applichi se stesso a codesto giudizio mediante una forma appresa più alta. $E$ questo non può essere altro che la nozione stessa di bene o di cosa conveniente con la quale si può giudicare di ciascun bene determinato. Perciò possono muovere se stessi a giudicare quegli esseri che conoscono la ragione universale di bene. E questi sono solo gli esseri intelligenti ${ }^{28}$.

In primo luogo si evidenzia che il movente principale dell'atto volitivo è una ratio, ed è precisamente la ragione di bene e di conveniente presente in un bene particolare ${ }^{29}$. In virtù della facoltà intellettiva - viene qui ribadito - l'agente razionale conosce il bene nella sua stessa ragione $^{30}$, ed è a causa di questa stessa ragione che tutte le forme particolari a cui tale ragione si applica sono principi moventi e che l'agente può muoversi verso tutti. Da notare infatti che la forma appresa, cui si riferisce l'Aquinate all'inizio della citazione, non è una forma universale, è invece una forma particolare, conosciuta sotto una ragione universale.

Per l'agente intellettuale la ratio principale della cosa da fare sarebbe il fine dell'azione stessa; e poiché egli non conosce solo il fine come cosa da raggiungere, ma anche la ragione di fine, vede che in questa ragione - questa forma universale - rientrano tante forme o ragioni particolari, ossia tanti modi di pervenire allo stesso fine. E quindi, una volta voluto il fine, può essere mosso da ognuna di queste forme - in cui si presentano dei beni particolari- in quanto tutte sono ordinate al fine; tutte partecipano della desiderabilità del fine voluto, e quindi della ragione di bene e di cosa conveniente ${ }^{31}$. Le molteplici forme che sono diverse e anche opposte, hanno dunque il potere di causare l'atto volitivo proprio in quanto partecipano tutte della stessa rationem finis.

Sembra quindi chiaro che per Tommaso, il bene particolare oggetto dell'inclinazione, presente nell'agente in virtù della forma, ha il potere di muovere la volontà alla scelta, in quanto la forma della scelta non è altro che la ragione del suo oggetto, il quale (essendo mezzo al fine) partecipa della desiderabilità del fine già voluto. Il testo di Contra gentiles è esplicito nell'affermare la forma particolare come principio movente: la forma appresa, per essere principio movente, necessita di partecipare della ragione stessa di bene e di conveniente, ossia di una

28 C.G., L. II, c. 48, § 3: «forma apprehensa est principium movens secundum quod apprehenditur sub ratione boni vel convenientis: actio enim exterior in moventibus seipsa procedit ex iudicio quo iudicatur aliquid esse bonum vel conveniens per formam praedictam. Si igitur iudicans ad iudicandum seipsum moveat, oportet quod per aliquam altiorem formam apprehensam se moveat ad iudicandum. Quae quidem esse non potest nisi ipsa ratio boni vel convenientis, per quam de quolibet determinato bono vel convenienti iudicatur. Illa igitur sola se ad iudicandum movent quae communem boni vel convenientis rationem apprehendunt. Haec autem sunt sola intellectualia).

29 Qui, come nel resto della trattazione, ci si riferisce indistintamente ad ogni cosa che si «presenti» come bene, quindi anche il bene apparente.

30 Cfr. S. Th., I, q. 82 a. 3: «nam obiectum intellectus est ipsa ratio boni appetibilis; bonum autem appetibile, cuius ratio est in intellectu, est obiectum voluntatis».

31 Cfr. De malo, q. 6 a. un. 
forma più alta; e proprio in virtù di detta partecipazione, essa stessa, anche se particolare, ha il potere di muovere, e quindi di inclinare all'azione. Infatti, si spiegava anteriormente, se l'agente possiede una determinata forma per una determinata azione, per ciò stesso è già inclinato ad agire.

Ora, entrando adesso nello specifico del nostro studio, va sottolineato che per il darsi dell'atto di scelta non basta che ci sia un desiderio di qualche cosa per il fine (un giudizio che dice «questo è buono»), ma deve essere il desiderio accompagnato dal discernimento di una cosa da un'altra ${ }^{32}$. La scelta consiste nella preferenza di una cosa rispetto all'altra che viene respinta ${ }^{33}$. Pertanto solo un giudizio di confronto dei beni tra loro può essere il giudizio previo alla scelta, e solo in esso si valuta e si presenta il meglio. Tutti sono beni ma soltanto nel confronto reciproco si vede se uno è migliore dell'altro e in chessenso lo è. Altrimenti se ogni bene venisse considerato unicamente in rapporto al fine, si avrebbe l'atto di consenso, che invece è previo alla scelta ${ }^{34}$. Perché si dia l'atto di scelta ciascun bene va confrontato anche con gli altri e quindi deve essere giudicato migliore rispetto agli altri. A motivo di ciò la scelta si pone al termine di un processo che ha per inizio la volizione del-fine, cui segue il lavoro deliberativo che si conclude poi in un giudizio ${ }^{35}$. Nella deliberazione infatti, l'intelletto paragona vari beni in rapporto al fine voluto e in rapporto tra loro, e formula così diversi giudizi di convenienza. Ciascuno di questi giudizi consiste nell'applicazione di una forma appresa a una cosa giudicata, e ciascuno presenta una ragione, principio movente per un'azione e quindi causa sufficiente per un atto di scelta. Al termine del processo deliberativo si arriva quindi a una visione articolata della situazione. E detta visione consisterebbe appunto nel giudizio aperto agli opposti: un atto intellettuale in cui simultaneamente si vedono le alternative eleggibili in rapporto al fine e in rapporto tra di loro.

Questo punto a mio avviso è centrale per la comprensione della scelta nonché della questione del meglio in Tommaso, come si vedrà in seguito. La libertà di scelta per l'Aquinate ha infatti la sua radice nel potere intellettuale di confrontare con l'universale la reale molteplicità del bene. Il fondamento metafisico della libertà è la natura molteplice del bene. Scrive infatti Tommaso:

la volontà non può volere una cosa, che sotto l'aspetto di bene. Ma poiché il bene è molteplice, essa non è necessariamente determinata a un oggetto ${ }^{36}$.

32 S. Th., I-II q. 13 a 2 ad 1: «non omnis appetitus alicuius propter finem, vocatur electio: sed cum quadam discretione unius ab altero».

33 Cfr. S. Th., I-II q. 13 a. 2 c.

34 Il bene particolare messo in confronto al fine e quindi giudicato adeguato a esso è infatti propriamente oggetto dell'atto di consenso.

35 Cfr. S. Th., I-II q. 14 a. 6.

$36 S$. Th. I q. 82 a. 2 ad 1: «Voluntas in nihil potest tendere nisi sub ratione boni. Sed quia bonum est multiplex, propter hoc non ex necessitate determinatur ad unum»s. Il giudizio intellettuale è apertura a una molteplicità. Il motivo principale dell'indeterminazione volitiva, offerto dall'Aquinate, non è costituito da un deficit della facoltà intellettuale, bensì dalla varietà multiforme del bene. L'intelletto coglie detta ricchezza e così può formulare un giudizio aperto: si presentano invero vari beni conosciuti come tali e quindi tutti sceglibili. Il giudizio è aperto nel senso che consiste in una formulazione non definitiva o non assolutamente conclusiva sul piano dell'azione. Presenta alla volontà diverse possibilità di azione: vale a dire, alla domanda «che cosa farò» offre una gamma di risposte tra cui la volontà può scegliere quella decisiva per l'azione. Non sembra quindi perfettamente rispondente alla posizione di Tommaso l'interpretazione di chi considera il giudizio pratico un mero giudizio di probabilità dovuto sempre a una mancata evidenza. Si legge ad es. in LEBACQZ J., Libre arbitre et jugement, Descleè de Brouwer, Paris-Bruges 1960, pp.134-137: «Percependo differenti mezzi per raggiungere un bene desiderato, il soggetto contraddirebbe la sua aspirazione a questo bene se non tentasse di determinare quale di questi mezzi sia il più adatto a farglielo raggiungere. Quale sarà il risultato di questa ricerca? Basandosi una volta di più sul carattere spazio-temporale degli oggetti dell'attività umana, si può immediatamente rispondere che non si tratterà mai di una evidenza 
Ora, tale reale molteplicità del bene può essere confrontata in quanto appartiene a un'unità e si dà in essa. Si legge ancora in Tommaso:

alla natura corrisponde sempre una determinazione unica senza alternative, proporzionata però alla natura in parole. Alla natura in genere corrisponde un determinato genere; alla natura presa come specie corrisponde un'unità di specie, alla natura individuale corrisponde un'unità individuale. Ma essendo la volontà una facoltà immateriale come l'intelletto ad essa corrisponde per natura un'unità di carattere universale (il bene), come l'unità di carattere universale, il vero, l'ente o la quiddità corrispondono all'intelletto. E il bene universale abbraccia la moltitudine dei beni particolari, verso i quali la volontà non è determinata ${ }^{37}$.

La facoltà intellettiva infatti -in virtù dell'immaterialità- ha invero la capacità di conoscere molte cose insieme in quanto formano un'unità, ossia servendosi di una sola specie intenzionale ${ }^{38}$; e questo è ciò che avviene propriamente nella formulazione del giudizio aperto agli opposti: si conosce la differenza e la comparazione di una cosa con un'altra —ossia di due termini- sotto l'aspetto di un'unica comparazione e un'unica differenza ${ }^{39}$ in rapporto al fine. La volontà invero, una volta voluto il fine, spinge la ragione a deliberare sui mezzi in vista del fine $^{40}$. Questa, deliberando sotto l'unica ragione del fine ${ }^{41}$-la ragione di bene e di cosa conveniente - attraverso il confronto e il paragone di vari beni particolari, perviene a una sorta di giudizio globale che raccoglie giudizi contrari. Tale giudizio quindi costituisce una causa della scelta: presenta diversi beni ognuno dei quali in se stesso è sufficiente a muovere la volontà.

Si rivela a questo punto difficile sostenere l'indifferenza volitiva come se la volontà non subisse alcuna attrazione rispetto ai beni particolari. Casomai, il problema sarebbe l'opposto:

propriamente detta. (...) Non cogliendo dell'universo che certi aspetti molto parziali, non penetrando nemmeno la natura della maggior parte degli esseri terrestri, lo spirito umano non può portare sul suo futuro che delle affermazioni approssimative e globali». In definitiva delle probabilità «che non restano altro che semplici probabilità» di modo che la differenza tra il momento della preacceptatio e quello del giudizio si riduce quasi alla distinzione tra una certezza (della volontà) e una incertezza (della ragione): «per natura l'atto di volontà implica sempre l'affermazione ferma di una proposizione che l'atto di conoscenza aveva posto soltanto come probabilen. Nonostante si riscontri una parte di verità in tali considerazioni, necessiterebbe di una ulteriore precisazione. Se invero il giudizio pratico offre solo una possibilità di azione — che diventa effettiva in virtù dell'atto di scelta — ciò non significa che l'intelletto non possa mai pervenire a una verità pratica. Tale interpretazione peraltro suggerisce alcune conclusioni che non rispondono alla visione del reale di Tommaso: sembrerebbe infatti che sempre, tra i molti beni esistenti, ce ne debba essere uno che sia migliore in senso assoluto a cui però la ragione non può pervenire perché è limitata. Se così fosse però la libertà umana sarebbe unicamente una conseguenza del limite dell'intelletto e allo stesso tempo Dio (Intelligenza perfetta) non sarebbe libero (il che non è in accordo con la posizione di Tommaso al riguardo). Si ritiene invece che l'affermazione dell'Aquinate rispetto alla molteplicità del bene faccia riferimento a molteplici possibilità di pervenire al Bene, tutte valide. L'apertura del giudizio consiste quindi nel potere conoscitivo di adeguazione e confronto della molteplicità del bene. La libertà deriva innanzitutto da un potere e non da un limite.

37 S. Th., I-II q. 10 a. 1 ad 3: «ad tertium dicendum quod naturae semper respondet unum, proportionatum tamen naturae. naturae enim in genere, respondet aliquid unum in genere; et naturae in specie acceptze, respondet unum in specie; naturae autem individuatae respondet aliquid unum individuale. Cum igitur voluntas sit quaedam vis immaterialis sicut et intellectus, respondet sibi naturaliter aliquod unum commune, scilicet bonum, sicut etiam intellectui aliquod unum commune, scilicet verum, vel ens, vel quod quid est. Sub bono autem communi multa particularia bona continentur, ad quorum nullum voluntas determinatur».

38 Cfr. S. Th., I, q. 85 a. 4: «...intellectus quidem potest multa intelligere per modum unius (...). Quaecumque ergo intellectus potest intelligere sub una specie, simul intelligere potest».

39 Cfr. S. Th., I, q. 85 a. 4, ad 4: «quando intellectus intelligit differentiam vel comparationem unius ad alterum, cognoscit utrumque differentium vel comparationem sub ratione ipsius comparationis vel differentiae».

40 Cfr. S. Th., I-II, q. 14 a. 1 ad 1.

41 Ci si riferisce qui a qualunque fine, anche subordinato, e non necessariamente al fine ultimo. 
tutti i beni particolari attirano. Tutti i mezzi che portano al fine ${ }^{42}$ hanno effettivo potere causale. Come può la volontà determinarsi a uno piuttosto che a un altro? Come si spiega infatti la determinazione volitiva verso un bene e non verso l'altro, e in particolare, la scelta del meglio? Infatti, se tutti i beni hanno equivalente potere per causare l'atto, come si può dare un meglio tra di essi?

Ma precisamente qui si entra nello specifico dell'atto di scelta per Tommaso. La scelta si accennava, è una preferenza, e perché si dia l'atto di scelta ciascun bene va confrontato anche con gli altri e quindi deve essere giudicato migliore rispetto agli altri. La domanda che si ripropone è quindi: se un bene si giudica migliore rispetto agli altri, in che senso ci può essere una scelta libera? Se infatti c'è un meglio significa che si dà gradualità nel bene, e laddove c'è gradualità non c'è libertà: se infatti un bene si presenta al soggetto come maggiore rispetto agli altri, lo prenderà necessariamente, e non ci sarà un equivalente potere causale di questo con gli altri che nel confronto risultano minori.

\section{LA SCELTA DEL MEGLIO}

Le domande trovano risposta solo a un livello più fondamentale: la considerazione della natura analogica del bene. La nozione di bene, e quindi anche di meglio, nella concezione di Tommaso non è una nozione univoca. Vale a dire, il bene non è solo molteplice in senso quantitativo (ossia vi sono tante forme in cui il bene si realizza), ma ciò in cui consiste l'essere bene o l'essere meglio di una cosa, è diverso rispetto a ciò in cui lo stesso consiste in un'altra. Come si è già visto precedentemente ${ }^{43}$, la comunanza del bene non è quella di una specie o di un genere. Il bene cioè non è univoco.

Risulta piuttosto eloquente per illustrare la molteplice diversità delle forme di bene, la molteplicità di esempi apportati da Tommaso nel commento all'Etica di Aristotele: il bene appartiene a tutte le categorie, e per ciascuna consiste in qualcosa di diverso rispetto a ciò in cui consiste per un'altra. Infatti,

di quelle cose di cui non vi è una ragione comune non ci può essere un'idea. Ma delle diverse categorie non vi è una ragione comune. Niente infatti di essi si predica in modo univoco. $\mathrm{E}$ il bene come l'ente poiché si converte con esso, si trova in qualsiasi categoria. Così nel che cos'è, cioè nella sostanza, bene si dice di Dio in cui non vi è malizia, $\mathrm{e}$ dell'intelletto, che è sempre retto. Nella qualità invece si dice della virtù che rende buono chi la possiede. Nella quantità si predica di ciò che è proporzionato, che è buono in quanto soggiace a misura. Nella relazione è buono ciò che è utile, che è buono in relazione al fine dovuto. Nel quando si dice del tempo opportuno, e nel dove del luogo adatto a camminare come una dimora. E lo stesso accade per gli altri generi. È evidente quindi che non c'è qualche cosa di buono che sia l'idea o la ragione comune di tutte le cose buone: altrimenti accadrebbe che il bene non si troverebbe in tutte le categorie, ma in una sola ${ }^{44}$.

42 Perché ci sia scelta infatti ci deve essere una pluralità di mezzi. Cfr. S. Th., I q. 13 a. 2: «cum electio sit preacceptatio unius respectu alterius, necesse est quod electio sit respectu plurium quae elegi possunt».

43 Cfr. $S$. Th., I-II q. 10 a. 1 ad 3.

44 In Ethicorum, I, lect. $6 \$ 81$ : «...eorum quorum non est una ratio communis, non possit esse una idea. Sed diversorum praedicamentorum non est una ratio communis. Nihil enim univoce de his praedicatur. Bonum autem sicut et ens, cum convertatur cum eo, invenitur in quolibet praedicamento. Sicut in quodquidest, idest substantia, bonum dicitur Deus, in quo non cadit malitia, et intellectus, qui semper est rectus. In qualitate autem virtus, quae bonum facit habentem. In quantitate autem commensuratum, quod est bonum in quolibet quod subditur mensurae. In ad aliquid autem bonum quod est utile, quod est bonum relatum in debitum finem. In quando autem tempus, scilicet opportunum, et in ubi locus congruus ad ambulandum, sicut dieta. Et idem patet in aliis generibus. Manifestum est ergo, quod non est aliquid unum bonum, quod scilicet sit idea, vel ratio communis omnium bonorum: alioquin oporteret, quod bonum non inveniretur in omnibus praedicamentis, sed in uno solo». 
Ci sono poi diverse ragioni di bene anche all'interno di una stessa categoria, infatti si può osservare che ciò in cui consiste il «tempo opportuno» nell'ambito delle arti militari, è diverso da ciò in cui esso consiste nell'agricoltura o nella medicina ${ }^{45}$. Inoltre anche le cose che sono buone per se:sono molte in forma, e quindi differiscono tra loro non solo in virtù della propria ragione specifica, ossia della loro essenza, ma anche nel loro essere buone. Spiega infatti Tommaso che

L'onore (...) e la prudenza e il piacere hanno ragioni diverse non solo proprie, in quanto cioè la ragione di onore in quanto è onore, differisce dalla ragione di prudenza in quanto è prudenza; ma in quanto è buona. Non è infatti una la ragione di bontà in queste cose, né sono appetibili secondo la stessa ragione. Resta quindi che ciò che è buono per sé non è qualcosa di comune o un'idea comune di tutti i beni ${ }^{46}$. .

Sembra quindi chiaro che la'concezione del bene di Tommaso non consiste in un'unica ragione: il bene è analogico. E pertanto la diversità delle forme di bene non consisterà in una mera differenza di intensità di un'unica ragione: consiste invece in una diversità di ragioni.

Ora, tornando adesso al caso specifico dell'oggetto di scelta, qui si osserva che tante cose sono mezzi in quanto sono adeguate al fine, e quindi sono tutte buone perché hanno una proporzione a esso. In questo senso i diversi beni, in quanto tutti sono mezzi per il perseguimento del fine, si equivalgono, ossia hanno identico potere per attrarre la volontà. E quindi in questo senso si può affermare una certa indifferenza della volontà rispetto ad essi. Ma indifferenza non nel senso che è la volontà a conferire forza attrattiva ad essi, bensì in quanto tutti portano al fine e quindi tutti indifferentemente la attirano.

Poiché però il bene è analogico, i diversi mezzi considerati in se stessi, sono estremamente diversi: sono cose diverse, hanno nature diverse; ossia in ognuna il suo essere proporzionata al fine consiste in qualcosa di diverso, qualcosa che l'altra non è. E nel giudizio di confronto tra le diverse alternative emerge che ciascuna è meglio in un aspetto. Poiché non si dà mera differenza di intensità di un'unica ragione, le alternative sono di molte ragioni e alle volte di molti generi. È per questo che si deve e si può scegliere, e che la scelta è libera ${ }^{47}$. Se si trattasse infatti di una gradazione di intensità di un'unica ragione, le alternative non sarebbero effettivamente tali, perché sempre ci sarebbe un più e un meno, una sarebbe migliore e l'altra peggiore sotto lo stesso aspetto oppure sarebbero totalmente uguali e non ci sarebbe modo di decidere

45 Cfr. ibidem, § 82: «ad cuius evidentiam sciendum est, quod sicut Plato ponebat quod res extra animam existentes assequuntur formam generis vel speciei per hoc quod participant ideam, ita quod anima non cognoscit lapidem nisi per hoc quod participat ideam lapidis, ita anima illarum scientiam et cognitionem participat per hoc, quod ipsarum formae sive ideae in ipsa imprimuntur. Ex quo sequitur, quod omnium eorum, quae habent unam ideam, est una scientia. Si ergo omnium bonorum esset una idea, sequeretur quod omnia bona pertinerent ad considerationem unius scientiae. Hoc autem videmus esse falsum, etiam quantum ad bona quae sunt in uno praedicamento. Quod addit, ne aliquis diversificationes scientiarum attribuat diversitati praedicamentorum. Videmus autem; quod tempus congruum, considerat quidem in rebus bellicis militaris, in aegritudinibus autem medicinalis, in laboribus autem exercitativa. Relinquitur igitur quod non est una communis idea bonorum».

46 Ibidem, lect. 7 \$ 94: «Honor enim et prudentia et voluptas habent diversas rationes non solum proprias, prout scilicet ratio honoris, inquantum est honor, differt a ratione prudentiae inquantum est prudentia; sed inquantum est bona. Non enim est una ratio bonitatis in omnibus his, nec secundum eamdem rationem sunt appetibilia. Unde relinquitur; quod id quod dicunt per se bonum, non est aliquid commune, velut una idea communis omnium bonorumi.

47 L'unica «forma di bene» che non è oggetto di scelta, quando la si conosce così come essa è, è il Bene divino. Egli è fuori da ogni genere e categoria e principio di tutti i generi. Cfr. al proposito S. Th., I, q. 3 a. 5 e q. 4 a. 3 ad 2. Evidentemente - è un dato dì esperienza - anche la felicità non è oggetto di scelta, ma propriamente non si può dire che la felicità sia una «forma" di bene nel senso inteso qui. Di per sé infatti la felicità significa solo il raggiungimento della totalità del bene, senza però dire in che cosa consiste quella totalità o quale ne sia la natura o la forma in cui si trova. 
fra loro ${ }^{48}$. Nella scelta invece, ogni alternativa si vede come migliore in qualche modo dell'altra, a causa di una diversa ragione (una ragione che l'alternativa concorrente non ha).

Una esemplificazione può aiutare a chiarire: poniamo che devo comprarmi una casa. Ho trovato due offerte che potrebbero essere alla mia portata perché entrambe hanno il prezzo e le dimensioni che avevo preventivato: si tratta di un appartamento, e di una villetta. Solo che l'appartamento è in centro, e molto vicino al luogo dove lavoro, l'inconveniente è che essendo in una strada principale è anche rumoroso. La villetta invece, pur essendo in città, è molto più lontana dal lavoro. Ha però il vantaggio che è in una zona tranquilla, è silenziosa; e quindi risulta più confortevole.

'Come si può notare nel momento in cui devo scegliere tra le due alternative, sto confrontando gli aspetti per cui ciascuna emerge rispetto all'altra e si presenta come migliore, sulla base di diverse categorie. Il «dove» (vicino al posto di lavoro) dell'appartamento, di contro alla «qualità» (confortevole) della villa. Le bontà della alternative non sono direttamente commensurabili; stanno in categorie diverse. Per questo, non si può dire in modo assoluto quale alternativa sia il meglio. Ciascuna è migliore rispetto all'altra in un aspetto per cui l'altra è peggiore. Si potrebbe anche considerare il paragone all'interno di una stessa categoria, per es. la qualità: l'appartamento è bello, la villa è confortevole. Anche qui si rileva una pluralità di forme che non sono direttamente commensurabili: non si può infatti paragonare direttamente la bellezza con la comodità; e quindi si ha la base per una scelta libera. Non si dà gradualità tra le due cose, non c'è un meglio assoluto, ma una pluralità di realtà migliori, perché sono realtà di generi diversi. Alla possibile domanda: perché si sceglie una cosa e non un'altra? Perché -ritornando all'esempio precedente- si sceglie la villa e non l'appartamento? La risposta adeguata per l'Aquinate è: perché si è data la preferenza alla tranquillità piuttosto che alla vicinanza al lavoro. E ci si potrebbe ancora chiedere: ma non c'è bisogno di una ragione per preferire la tranquillità ${ }^{49}$ ? A me sembra che la risposta qui sarebbe: sì c'è bisogno di una ragione ma non deve essere una ragione diversa dalla tranquillità stessa. La tranquillità è ciò che rende migliore la villa; ma non ci vuole un'altra cosa per rendere migliore la tranquillità stessa, ossia la tranquillità è un bene in se stesso, di per sé, e non necessita di un ulteriore bene o principio che lo renda tale per essere scelto ${ }^{50}$. $\grave{E}$ bene in un senso in cui la vicinanza al lavoro non lo è, e quindi in questo senso è migliore (è più tranquillità!). Solo che pure la vicinanza è bene di per sé, e in un senso in cui la tranquillità non lo è, e in questo senso è migliore. Per questo si sarebbe potuto anche scegliere altrimenti. Esiste insomma una molteplicità di forme di bene, ognuna delle quali fornisce una sua misura di meglio o peggio.

L'oggetto di scelta presenta quindi una peculiarità: da una parte la scelta è possibile perché si dà confronto tra $i$ beni. Dall'altra i beni confrontati non sono direttamente confrontabili; c'è invero una sorta di incommensurabilità tra essi che a sua volta rende possibile la scelta. I beni oggetto di scelta non sono invero direttamente paragonabili tra di loro o comunque sono paragonabili ma solo fino a un certo punto. Si è osservato infatti che gli aspetti per cui si può scegliere una cosa o un'altra, considerati in se stessi non hanno nulla in comune nelle loro definizioni. Che cosa accomuna infatti la vicinanza al lavoro con la tranquillità? La comunanza è data solo da una certa analogia di proporzionalità che si potrebbe tradurre in questi termini: così come la vicinanza al lavoro dell'appartamento favorisce il lavoro, la tranquillità della villa

\section{Cfr. I-II q. 13 a. 6 ob. 3.}

49 Questa è la domanda che pone Colleen Mc CLUSKEY a Tommaso in un recente articolo sulla libertà di scelta: Intellective Appetite and the Freedom of Human Action, The Thomist, 66 (2002), pp. 421 -456. L'autrice rinviene una lacuna nella spiegazione tomista della libertà di scelta perché lascerebbe irrisolta tale questione.

50 Eloquente al proposito quanto scrive Tommaso in S. Th., I q. 5 a. 5 ad 2: «ad secundum dicendum quod modus, species et ordo eo modo dicuntur bona, sicut et entia, non quia ipsa sint quasi subsistentia, sed quia eis alia sunt et entia et bona. Unde non oportet quod ipsa habeant aliqua alia, quibus sint bona. Non enim sic dicuntur bona, quasi formaliter aliis sint bona; sed quia ipsis formaliter aliqua sunt bona; sicut albedo non dicitur ens quia ipsa aliquo sit, sed quia ipsa aliquid est secundum quid, scilicet album». 
favorisce la vita in casa ${ }^{51}$. Come si vede si tratta di una comunanza estremamente debole. E comunque questa analogia - l'analogia del bene — spiega in che modo si possono vedere insieme, sotto un'unica specie intelligibile - come beni- e allo stesso tempo non vederli come due intensità della stessa forma. Sono forme irriducibilmente diverse e quindi anche criteri irriducibili di scelta. Ma se si possono paragonare in qualche modo è perché esiste una qualche unità. E l'unità è data dal bene più universale, in questo caso il bene totale del soggetto al quale appartengono molti sensi di bene, e in cui pertanto possono rientrare cose diverse e anche incommensurabili tra loro se considerate solo in se stesse.

Ed è qui a mio parere che emerge il ruolo essenziale dell'intelletto nella libertà di scelta. Un ruolo strutturalmente inscindibile dalla causalità del bene e dalla sua natura analogica. Infatti se le cose diverse e molteplici che sono oggetto di scelta, in se stesse non hanno pressoché nulla in comune tra loro, solo in virtù della facoltà intellettiva si possono cogliere le relazioni e si può operare quel confronto necessario per scegliere e perché la scelta sia libera. $\mathrm{E}$ tale confronto è ciò che si realizza nel giudizio aperto agli opposti: un ultimo giudizio che presenta diverse alternative tutte eleggibili, perché tutte in modo diverso, secondo diverse proporzioni, sono convenienti al fine ${ }^{52}$. Tale giudizio contiene quindi in sé, sia il principio - il fine da perseguire-, che le conclusioni - i giudizi diversi e opposti che presentano un'opinione ragionevole per il perseguimento del fine, a favore di una cosa da fare, e un'opinione ragionevole contro quella cosa da fare e a favore di un'altra ${ }^{53}$.

Mi sembra quindi si possa sostenere che il giudizio cui termina l'analisi deliberativa e che precede immediatamente la scelta sia precisamente un giudizio aperto agli opposti ${ }^{54}$. Infatti, alla luce di quanto affermato, si può asserire che questo giudizio sia al contempo causa dell'atto di scelta —offre una ragione sufficiente per spiegare l'atto di scelta—, e della sua libertà —non la muove necessariamente secondo una ragione-.

In questo giudizio infatti sono contenute diverse forme o ragioni, le quali sono le cause di possibili scelte diverse (ogni forma o ragione è causa per una scelta); in tal senso si può dire

51 Su questo punto si veda FLANNERY K., Acts Amid Precepts, The Aristotelian Logical Structure of Thomas Aquinas's Moral Theory, The Catholic University of America Press, Washington 2001, pp. 97-100.

52 Si preferisce la denominazione di giudizio globale o giudizio aperto agli opposti, evitando invece la denominazione di ultimo giudizio pratico che potrebbe confondersi con altri giudizi che hanno lo stesso nome ma che non coincidono con questo. Nemmeno si userà qui il termine «giudizio indifferente», in quanto ritenuto non corretto ed equivoco. Potrebbe invero dar adito a pensare che in questo giudizio le diverse possibilità offerte per la scelta siano uguali, cosa che non è; si tratta invece di possibilità diverse ma con lo stesso potere causale: tutte conducono ugualmente al fine, ma tutte vi conducono in modo differente.

53 Al proposito va rilevato che, anche se nella maggior parte dei casi è così, non sempre la deliberazione rispetto a un unico fine particolare, porta a varie alternative eleggibili. Dato un certo fine particolare infatti, potrebbe darsi il caso che lo si possa conseguire solo con un mezzo; e pertanto questo mezzo sarebbe davvero necessario per il fine. In questo caso non ci sarebbero alternative. Ma poiché l'agente può deliberare anche sui fini particolari (in quanto particolari), la libertà di scelta resta. Si può invero considerare quel mezzo anche sotto un aspetto che è diverso da quello che lo rende necessario (ponendosi un altro fine particolare, diverso e forse opposto al precedente) e che rende in qualche modo conveniente - almeno in apparenza- - il non prenderlo.

54 Cfr: $S$. Th., I q. 83 a. 1: «Sed homo agit iudicio, quia per vim cognoscitivam iudicat aliquid esse fugiendum vel prosequendum. sed quia iudicium istud non est ex naturali instinctu in particulari operabili, sed ex collatione quadam rationis; ideo agit libero iudicio, potens in diversa ferri. Ratio enim circa contingentia habet viam ad opposita; ut patet in dialecticis syllogismis, et rhetoricis persuasionibus. Particularia autem operabilia sunt quaedam contingentia, et ideo circa ea iudicium rationis ad diversa se habet, et non est determinatum ad unum. Et pro tanto necesse est quod homo sit liberi arbitrii, ex hoc ipso quod rationalis est's. Faccio presente che a proposito del giudizio che precede l'atto di scelta in ambito tomista ci sono interpretazioni divergenti. Non voglio entrare qui nella discussione dell'argomento, $\mathrm{mi}$ limito solo ad accennare che la linea interpretativa inizialmente menzionata (cfr. nota 2 del presente articolo) ritiene che il giudizio aperto agli opposti non sia sufficiente a causare l'atto volitivo. Per il darsi effettivo dell'atto ci deve essere un giudizio già determinato dalla volontà cui seguirà la scelta. Per una discussione di tale posizione rimando al mio studio La libertà e la razionalità della scelta in Tommaso d'Aquino, EDUSC, Roma 2002. 
che il giudizio che le contiene tutte, è causa sufficiente di ogni scelta. Allo stesso tempo, in questo giudizio, l'agente intellettuale vede simultaneamente le conclusioni in rapporto tra di loro e con il principio; e quindi ogni conclusione viene vista come contingente. L'evento sillogistico che ha portato al giudizio non è consistito in una dimostrazione. Le conclusioni del sillogismo pratico derivano dalle premesse e trovano in esse la loro causa, ma non ne derivano in modo necessario ${ }^{55}$. Le premesse potrebbero avere anche un'altra conclusione, perché non tutto ciò che è ordinato al fine, è indispensabile per il suo raggiungimento ${ }^{56}$. E il giudizio aperto agli opposti presenta tutto questo: si vede quindi che il principio (il fine) non esige necessariamente quella conclusione (quel mezzo) perché contemporaneamente si vede anche un'altra conclusione e quindi che un altro mezzo può essere scelto, ossia che anch'esso ha un potere sufficiente per causare un atto di scelta. Poiché quindi entrambi i mezzi hanno potere causale ciascuno fa da impedimento all'altro, e nessuno causa necessariamente l'atto. Nella concezione di Tommaso infatti non ogni causa sufficiente è necessaria. Ci sono invero cause che sono sufficienti a produrre un effetto, ma possono esserne impedite, da agenti esterni o interni; e, nel caso della volontà, dalla volontà stessa, come si vedrà ${ }^{57}$. Tali cause sono le cosiddette cause contingenti.

Il bene particolare allora, pur muovendo, ossia pur suscitando il desiderio dell'agente vo- litivo, non muove in modo necessario, cioc̀ non determina necessariamente l'atto dell'agente. E questo perché ci sono altri beni che ne hanno il potere, e che, presentandosi in simultanea, fanno da impedimento alla volizione necessaria di un unico bene particolare.

È la volontà quindi che non essendo determinata a nessun bene in particolare, si deve autodeterminare verso uno dei beni particolari che l'intelletto le presenta come tali. Ossia, in virtù della volizione del fine, e del confronto tra i diversi beni con il fine e tra di loro, liberamente si porta su uno o sull'altro in base all' aspetto migliore in esso considerato ${ }^{58}$. E si può anche aggiungere che se da una parte $\mathrm{i}$ beni impediscono rispettivamente la volizione necessaria di uno di essi, è la volontà in ultima istanza che pone gli stessi impedimenti e che è libera di porli: quando considera i due beni ma usa la considerazione di uno per far sì che esso muova il suo desiderio e impedisca all'altro di muoverlo, cioè lo sceglie. Interessante notare che nel mo-

55 La natura del sillogismo pratico viene ben illustrata da FLANNERY (2001), pp. 8-12, il quale fornisce altresì riferimenti bibliografici interessanti sugli studi della più recente letteratura analitica al riguardo. A illustrare il punto di cui sopra si legge a p. 10: «"Un altro aspetto peculiare del sillogismo pratico è che non esige (cioè non necessita) la propria conclusione. Supponiamo che il ragionamento pratico di qualcuno consista in questo (abbreviato) sillogismo pratico: «ho bisogno di coprirmi; un mantello serve a coprirmi; prendo un mantello". Ma supponiamo anche che tutti i mantelli siano infettati da insetti velenosi. Praticamente parlando, uno non è obbligato a prendere un mantello. Può, se vuole, prendere una coperta (presumendo che le coperte non siano infettate). Questa caratteristica del sillogismo pratico è spesso chiamata «revocabilità»: la conclusione di un sillogismo pratico è revocabile per fattori estranei». Anche ibidem, p. 131: «Il fine di un sillogismo pratico non è trovare una verità ma arrivare da qualche parte - e ci sono vari modi di farlo, nessuno dei quali, presumibilmente', logicamente vincolante. Perciò, c'è una sorta di distanza pratica fra quello che si pensa di fare in generale e ciò che si fa in realtà. E' in questo spazio tra l'universale e il particolare che Tommaso colloca il libero arbitrio". Sullo stesso tema si veda anche DEWAN L., O.P., St. Thomas and the Causes of Free Choice, «Acta Philosophica», 8 (1999), pp. 90-91 e p. 95.

56 Cfr. S. Th., I-II, q. 13 a. 6 ad 1: «ad primum ergo dicendum quod non semper ex principiis ex necessitate procedit conclusio, sed tunc solum quando principia non possunt esse vera si conclusio non sit vera. Et similiter non oportet quod semper ex fine insit homini necessitas ad eligendum ea quae sunt ad finem, quia non omne quod est ad finem, tale est ut sine eo finis haberi non possit; aut, si tale sit, non semper sub tali ratione consideratur?.

57 Interessante in Tommaso la nozione di impedimento per la spiegazione della causalità contingente. Cfr. De malo, q. 6 a un. ad 15: «non omnis causa ex necessitate inducit effectum, etiam si sit causa sufficiens eo quod causa potest impediri, ut quandoque effectum suum non consequatur; sicut causae naturales, quae non ex necessitate producunt suos effectus, sed ut in pluribus, quia in paucioribus impediuntur. Sic ergo illa causa quae facit voluntatem aliquid velle, non oportet quod ex necessitate hoc faciat: quia potest per ipsam voluntatem impedimentum praestari, vel removendo talem considerationem quae inducit eum ad volendum, vel considerando oppositum, scilicet quod hoc quod proponitur ut bonum secundum aliquid non est bonums.

58 Cfr. S. Th., I-II q. I3 a. 6 ad 3. 
mento della scelta non si perde di vista la bontà dell'alternativa respinta. Pur vedendo che la cosa si presenta come buona, si considera anche che in qualche modo non è buona ${ }^{59}$.

E se l'intelletto è il fondamento psicologico della libertà di scelta della volontà, la natura del bene ne è il primo fondamento metafisico. Il bene è vario, molteplice, diverso ${ }^{60}$. E per questo si può esercitare la libertà di scelta, e si può scegliere liberamente il meglio. Non si dà un meglio assoluto (non c'è un perché determinante); ogni alternativa si offre come migliore in qualche aspetto; e in quell'aspetto specifico in cui una è migliore, l'altra alternativa è effettivamente peggiore; ma quest'ultima si può presentare come alternativa proprio in quanto ha un altro aspetto per cui è migliore. È quindi in virtù di un meglio che la volontà decide. Detto meglio se non la vincola necessariamente a sé, in quanto non è assoluto, offre comunque una motivazione all'atto.

Si può concludere allora che la scelta è libera (nel duplice senso di non necessitata e dominante), e ragionevole. È la volontà che decide quale cosa prendere; ma il motivo per cui prenderla lo fornisce il bene stesso. Non è quindi la volontà con la sua scelta a conferire l'aspetto di bontà (o la capacità attrattiva) alla cosa.

Federica Bergamino

Pontificia Università della Santa Croce

Roma (Italia)

f.bergamino@usc.urbe.it

59 Questo si evidenzia molto bene in de malo, q. 6 a. un. ad 15 quando si legge: «quia potest per ipsam voluntatem impedimentum praestari, vel removendo talem considerationem quae inducit eum ad volendum, vel considerando oppositum, scilicet quod hoc quod proponitur ut bonum secundum aliquid non est bonum».

60 È la varietà del bene che spiega anche la scelta del male per Tommaso. Si legge infatti in un'obiezione di de malo q. 6 a. un. ob. 6: «nullius potentiae actus potest esse nisi in suum obiectum; sicut visus actio non potest esse nisi circa visibile. sed obiectum voluntatis est bonum. Ergo voluntas non potest velle nisi bonum. Ex necessitate ergo vult bonum, et non habet liberam electionem boni vel mali». Cui segue la risposta: "Ad sextum dicendum, quod ex hoc quod bonum est obiectum voluntatis, potest haberi quod voluntas nihil velit nisi sub ratione boni. sed quia sub ratione boni multa et diversa continentur, non potest ex hoc haberi quod ex necessitate voluntas moveatur in hoc vel illud». 